\title{
Hyaluronan content of Wharton's jelly in healthy and Down syndrome fetuses
}

\author{
Luigi Raio ${ }^{\mathrm{a}, *}$, Antonella Cromi ${ }^{\mathrm{b}}$, Fabio Ghezzi ${ }^{\mathrm{b}}$, Alberto Passi ${ }^{\mathrm{c}}$, Evgenia Karousou ${ }^{\mathrm{c}}$, \\ Manuela Viola ${ }^{\mathrm{c}}$, Davide Vigetti ${ }^{\mathrm{c}}$, Giancarlo De Luca ${ }^{\mathrm{c}}$, Pierfrancesco Bolis \\ a Department of Obstetrics and Gynecology, University of Berne, Effingerstrasse 102, 3010 Berne, Switzerland \\ ${ }^{\mathrm{b}}$ Department of Obstetrics and Gynecology, University of Insubria, Varese, Italy \\ ${ }^{\mathrm{c}}$ Department of Experimental and Clinical Biomedical Sciences, University of Insubria, Varese, Italy
}

Received 18 July 2004; received in revised form 24 October 2004; accepted 24 January 2005

\begin{abstract}
The mechanisms by which the excess genetic material of chromosome 21 results in the dysmorphologic features of Down syndrome (DS) are largely unknown. It has been found that the extracellular matrix of nuchal skin of DS fetuses exhibits an higher content of hyaluronan (HA) compared to that of euploid fetuses. Since HA plays a central role in many morphogenetic processes during embryogenesis, an alteration in its metabolism could be involved in the pathogenesis of several structural defects of DS. The extracellular matrix of umbilical cord (UC) is the mammalian tissue with one of the highest content of HA. Therefore we sought to explore the quantitative HA modifications during gestation, tissue distribution and HA metabolism in euploid and DS UCs. Euploid UCs ( $n=28)$ and UCs from DS fetuses $(n=13)$ were obtained after termination of pregnancy, spontaneous abortion, or at delivery. Quantitative and molecular size analysis were performed using HPLC and FPLC. Tissue distribution was visualized by immunohistochemistry. Gene expression for HA synthases (HAS) and hyaluronidases (HYAL) were quantified by real-time PCR techniques and HYAL activity was detected by zymography. In euploid UC only HA of a molecular weight of $1700 \mathrm{kDA}$ was present while in DS UC an additional lower weight HA molecule of $1100 \mathrm{kDA}$ was found. Immunohistochemistry showed a larger amount of Wharton's jelly HA in DS UCs than in euploid UC. Real-time PCR analysis showed that HAS 2 and HYAL 2 were expressed at significant levels in all specimens. A higher expression of HAS 2 and a lower expression of HYAL 2 was found in the Wharton's jelly of DS fetuses compared to that of euploid fetuses at 14 weeks of gestation. On the contrary, at term HYAL 2 expression was higher in DS specimens than in those from euploid fetuses. Zymographic studies showed a similar behavior with a lower HYAL activity at early gestation and a higher HYAL activity at term gestation in DS UCs compared to euploid specimens. Therefore we can conclude that HA is more represented in DS UCs than in euploid UCs. A complex alteration of the HA metabolism characterized by an increased synthesis of lower weight HA molecules is a peculiarity of DS UCs.
\end{abstract}

(C) 2005 Elsevier B.V./International Society of Matrix Biology. All rights reserved.

Keywords: Umbilical cord; Down syndrome; Hyaluronan; Hyaluronidase; Hyaluronan synthase

\section{Introduction}

HA is a component of the connective tissue and is present in the extracellular matrix (ECM) of most vertebrate tissues (Laurent, 1998). In some cases HA is a major

\footnotetext{
* Corresponding author. Tel.: +41 03163210 10; fax: +41 0316321646.

E-mail address: luigi.raio@insel.ch (L. Raio).
}

constituent as, for example, in the vitreous of the human eye (0.1-0.4 mg/g wet weight) (Meyer and Palmer, 1934), or in the matrix produced by the cumulus cells around the oocyte prior to ovulation $(\sim 0.5 \mathrm{mg} / \mathrm{ml})$. (Salustri et al., 1990). In other tissues, such as cartilage (Knudson and Knudson, 2001), even representing less of the mass of the tissue, HA serves as an essential structural element in the matrix. In the past decade a growing body of evidence has shown that HA, previously thought of as an inert space-filling component of 


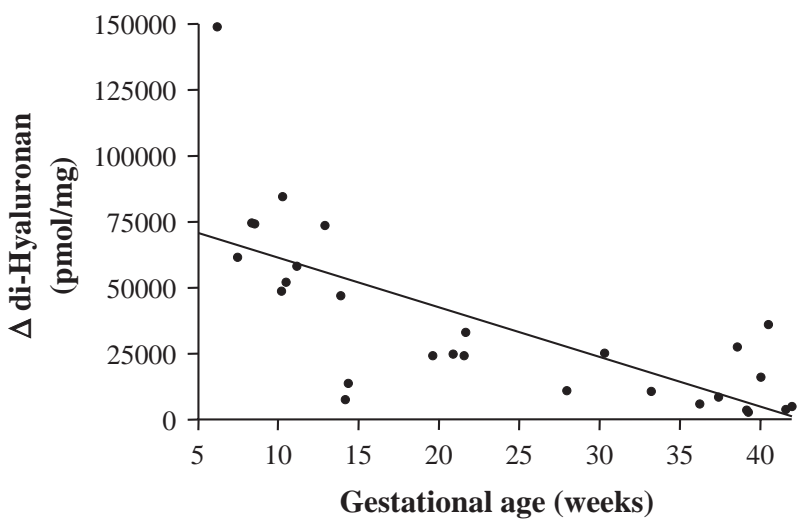

Fig. 1. Correlation between gestational age and HA content in umbilical cords of euploid fetuses [Spearman Rank Correlation: $r=-0.80, p<0.001$ (95\% CI: $-0.90 ;-0.60)]$. Data are expressed as pmoles of unsaturated disaccharides derivatized with AMAC in $1 \mathrm{mg}$ of dry tissue.

ECM, plays a central role in many morphogenetic processes during vertebrate embryogenesis.

Down syndrome represents a condition of inborn errors of morphogenesis that results from an extra copy of chromosome 21. The mechanisms by which the excess genetic material of chromosome 21 results in the dysmorphology of Down syndrome have not been defined. Several studies showed that alterations of the ECM, with a substantial increase in HA concentration, could be the underlying cause for the increased nuchal translucency frequently present in trisomy 21 fetuses at early gestational age (Von Kaisenberg et al., 2003; Bohlandt et al., 2000; Von Kaisenberg et al., 1998a; Brand-Saberi et al., 1994a,b). Moreover, the composition of the ECM seems critical for the development of heart defects involving endocardial cushion differentiation, in particular the atrioventricular canal, which accounts for nearly half of the congenital heart diseases observed in Down syndrome subjects (Eisenberg and Markwald, 1995).

The matrix of the umbilical cord is considered to be the mammalian tissue with one of the highest content of HA ( $\sim 4 \mathrm{mg} / \mathrm{ml}$ ) (Weissman and Meyer, 1954). Recently, it has been reported that the umbilical cord morphology of Down syndrome fetuses is different than that of healthy fetuses (Raio et al., 2004; Moessinger et al., 1986). In particular, it has been noted that trisomy 21 fetuses seem to have shorter umbilical cord as well as a lower number of coils than healthy fetuses (Raio et al., 2004; Moessinger et al., 1986). The histological and biochemical basis of these findings are largely unknown. The composition of ECM of umbilical cords in Down syndrome fetuses has been poorly investigated to date. We performed this study to explore the content, distribution pattern, and molecular size of hyaluronan in ECM of umbilical cords of euploid and Down syndrome fetuses. Moreover, the expression of genes encoding hyaluronan synthases (HAS) and hyaluronidases (HYAL) in umbilical cord ECM has been evaluated.

\section{Results}

The umbilical cord content of HA has been analysed measuring HA disaccharides by HPLC after specific enzymatic digestions (see Experimental procedure section for details). In euploid fetuses the umbilical cord HA amount decreased significantly with advancing gestation (Fig. 1). Similarly, an inverse significant correlation was found between HA concentration and gestational age in Down syndrome cases $[r=-0.75, p=0.003$ (95\% CI: $-0.92 ;-0.34)$ ]. Considering only specimens between 14 and 21 weeks of gestation (since most trisomy 21 UCs were achieved from terminations of pregnancy at this gestational age), an higher HA content was found in trisomy 21 fetuses $(n=10)$ compared with healthy fetuses $(n=7)$ [44840 (26010-71460) vs. 24370 (7729-47110) disaccharide $\mathrm{pmol} / \mathrm{mg}$ dry tissue; $p=0.009$ ] (Fig. 2). A gestational age-matched ( \pm 4 days) healthy control was available for 9 trisomy 21 fetuses. The umbilical cord HA content was higher in trisomy 21 fetuses than in healthy fetuses in 8 pairs (Fig. 3). The median difference in umbilical cord HA content between pairs was $9113 \mathrm{pmol} /$ mg dry tissue (range: - 16491-63728).

Immunohistochemistry of HA based on biotinylated HA binding protein (bHABP) decoration showed a different distribution pattern in umbilical cords from trisomy 21 fetuses compared to the control group (Fig. 4). As expected from biochemical analysis, Wharton's jelly of trisomic cords was intensely stained, showing a large amount of HA with homogeneous distribution, whereas euploid umbilical cord specimens were much less positive for HA. On the contrary, the intensity of HA decoration observed in smooth muscle cells of umbilical cord vessels was much higher in euploid than in trisomy 21 umbilical cords. This HA distribution pattern did not change comparing umbilical cord specimens at early and late gestational age both in normal and trisomy 21 fetuses.

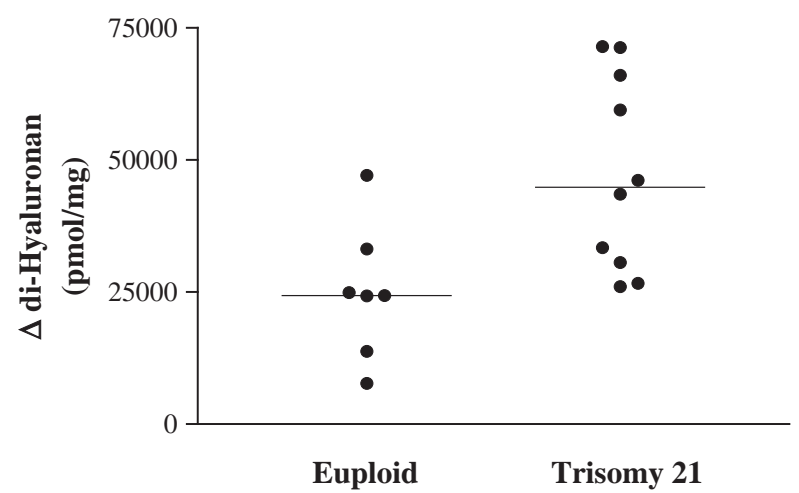

Fig. 2. Hyaluronan content in euploid and trisomy 21 umbilical cords between 14 and 21 weeks of gestation. Data are expressed as pmoles of unsaturated disaccharides derivatized with AMAC in $1 \mathrm{mg}$ of dry tissue. 


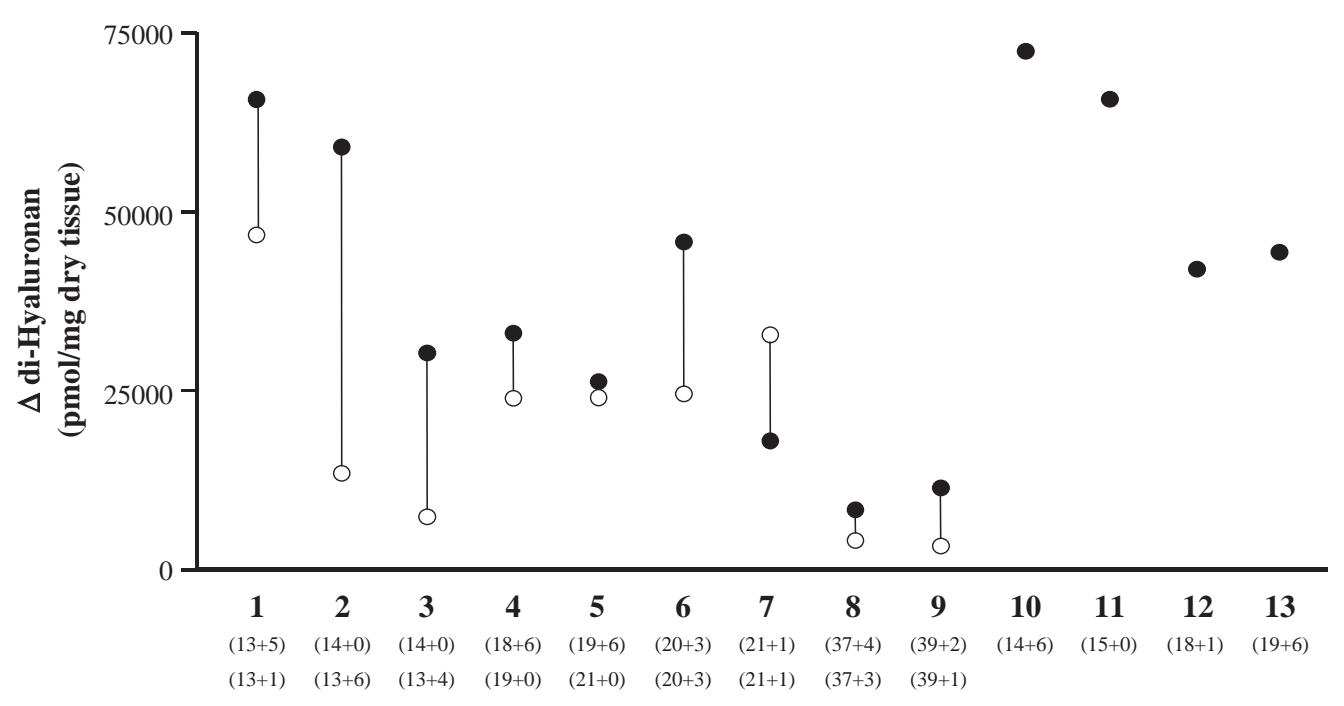

Gestational age-matched pairs

Fig. 3. Hyaluronan content in trisomy 21 and euploid umbilical cords matched for gestational age $( \pm 4$ days). A suitable control was not available for the last four cases of Down syndrome umbilical cords. (Ocontrol; $\bullet$ trisomy21). In parentheses is reported the gestational age, weeks + days.

Structural studies on the HA molecular size were carried out using a FPLC analysis, in order to separate intact mature HA molecule from its degradation fragments (oligosaccharides) (Fig. 5). Chromatographic data showed that the umbilical cord extracellular matrix of euploid fetuses at 14 weeks of gestation contains large HA molecules (about 1700 $\mathrm{kDA}$ ), included in a single peak, and the retained fraction further analysed by FACE (Fig. 5, panel A). Conversely, in trisomy 21 specimens of the same gestational age, FPLC analysis splitted mature HA in two chromatographic peaks (Fig. 5, panel B). In addition to the $1700 \mathrm{kDa}$ fraction, a population of HA with lower molecular weight (1100 kDA) has been observed. To check peak composition, analysis of the eluted material in the peaks was carried out with electrophoresis (FACE), analysing unsaturated disaccharides obtained after specific enzymatic treatment of the material eluted from the column and AMAC derivatization. These data showed that peaks eluted in void volume of the column
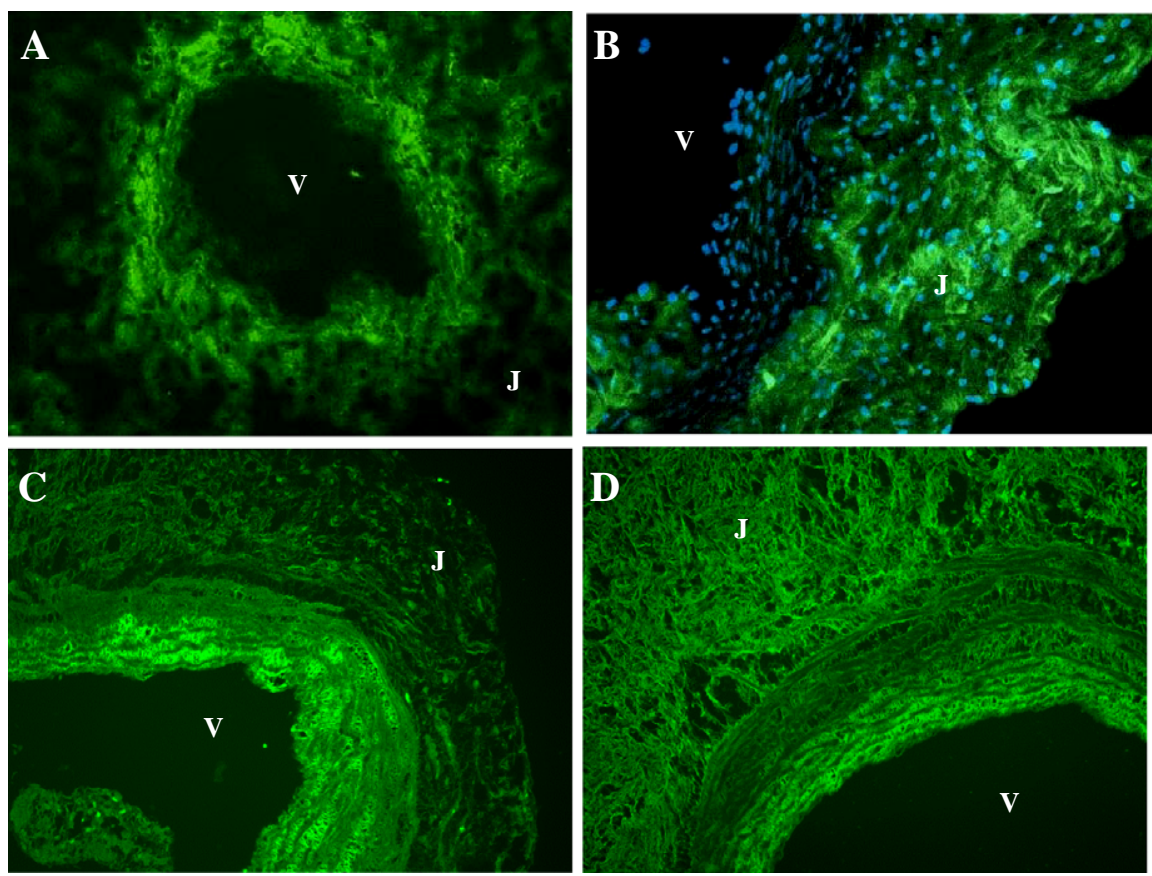

Fig. 4. Immunohistochemical staining for hyaluronan with biotin-labeled HA Binding Protein (bHABP). Magnification $\times 100$. (A) euploid umbilical cord at 13 weeks +4 days; (B) trisomy 21 umbilical cord at 14 weeks +0 days (stained additionally with DAPI to identify carefully the smooth muscle cells area); (C) euploid umbilical cord at 37 weeks +4 days; (D) trisomy 21 umbilical cord at 37 weeks +3 days. V, umbilical cord vessel; J, Wharton's jelly. 
(i.e. both peaks of 1700 and $1100 \mathrm{kDa}$ ) contain only HA, while all the other retained fractions contained sulphated oligosaccharides.

Real-time PCR experiments showed that only HAS 2 and HYAL 2 were expressed at significant levels in umbilical cords of either trisomy 21 and euploid fetuses, irrespective of gestational age, whereas HAS 1, HAS 3 and HYAL 1 were almost undetectable. Fig. 6 displays HAS 2 and HYAL 2 gene expression in umbilical cords obtained from two Down syndrome and two euploid fetuses at 14 weeks of gestation and at term. mRNA from Wharton's jelly and umbilical vessels was extracted separately only in term specimens. Indeed, in 14 weeks samples, the vessels portion was contaminated by Wharton's jelly tissue, and therefore we were able to purify only Wharton's jelly mRNA. In Down syndrome specimens HAS 2 expression in the Wharton's jelly was higher than in euploid specimens, irrespective of gestational age (Fig. 6, panel 1). Opposite findings were evident in umbilical vessels fraction in specimens at term (Fig. 6, panel 2). Interestingly, in Wharton's jelly of euploid specimens the HAS 2 expression decreases during pregnancy, whereas in Down syndrome UCs the expression of

\section{mAu}
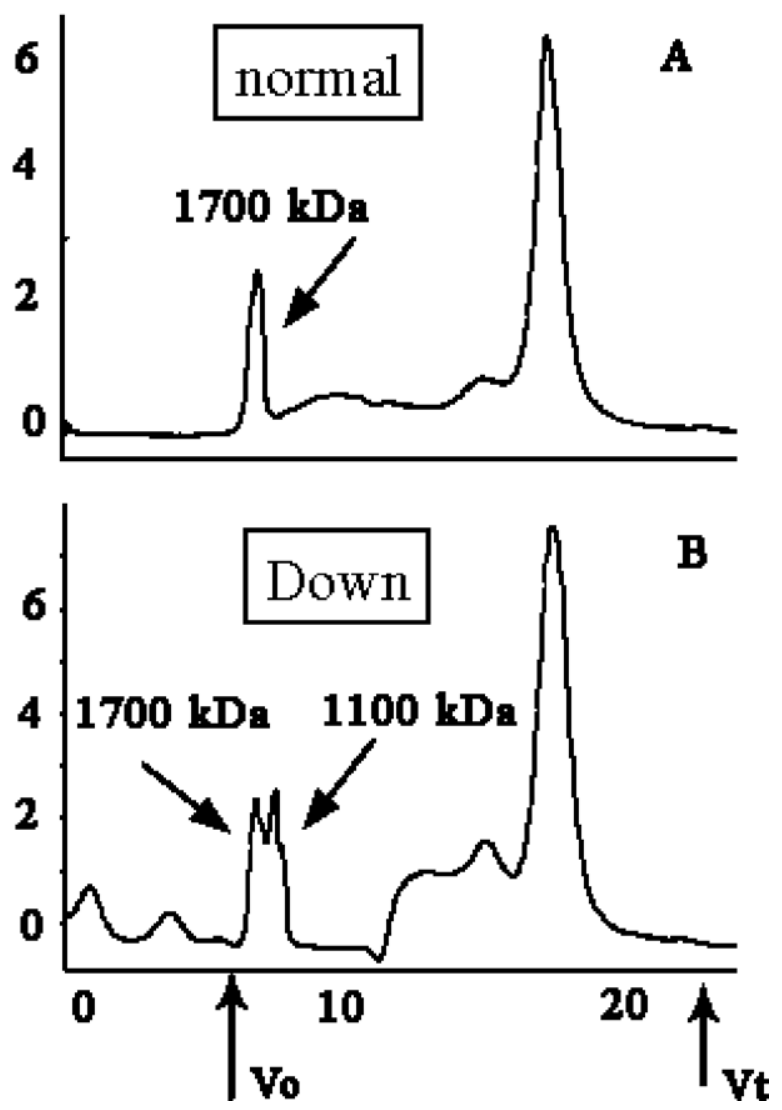

Fig. 5. Gel filtration chromatography of glycosaminoglycans extracted from human umbilical cords at 14 weeks of gestation. (A) material from euploid fetuses. (B) material from trisomy 21 specimens. The arrows indicate the calculated molecular mass of HA containing peaks.
HAS 2 showed an opposite trend (Fig. 6, panel 1). At 14 weeks of gestation, Wharton's jelly from euploid specimens showed an higher HYAL 2 expression than that in trisomy 21, whereas the trend is opposite at term of gestation (Fig. 6, panel 3). Similarly to HAS 2 data, in euploid tissues the HYAL 2 expression decreases from 14 weeks to term, whereas in Down syndrome tissues the trend is opposite (Fig. 6, panel 3). HYAL 2 expression in the umbilical vessels is much higher in trisomy 21 specimens than in normal tissue at term (Fig. 6, panel 4).

In Fig. 7 are shown the HYAL activities in samples from umbilical cords at 14 weeks and term using gel zymography. In gel zymography experiments, samples matching for gestational age and containing the same amount of protein were compared, in the range of 10 to 50 $\mu \mathrm{g}$. Gels were developed in buffers either at $\mathrm{pH} 3.5$ or 5 , but only in $\mathrm{pH} 3.5$ was the HYAL activity detectable. Two bands were mainly detected in all samples with molecular masses of 80 and $67 \mathrm{kDa}$. In addition, Down syndrome specimen at term gestation presented a third band of 51 $\mathrm{kDa}$. The molecular mass of the bands were measured using a mix of standard proteins running in the same gel. As expected from real-time PCR experiments, zymography confirmed that in the 14 weeks specimen the HYAL activity was more evident in euploid than in Down syndrome tissues (Fig. 7, lane 2 and 3 respectively), whereas at term the enzymatic activity is more detectable in Down syndrome samples in comparison to healthy specimen (Fig. 7, lane 4 and 5).

\section{Discussion}

The present study clearly demonstrates that umbilical cords of Down syndrome fetuses have a higher HA amount than euploid fetuses and a peculiar distribution pattern of this glycosaminoglycan. This finding is consistent with previous immunohistochemistry investigations on nuchal skin of trisomy 21 fetuses at term (Von Kaisenberg et al., 2003; Bohlandt et al., 2000; Von Kaisenberg et al., 1998a; Brand-Saberi et al., 1994a,b). An interesting finding of the present study is the progressive decrease with gestational age of HA content found in both normal and Down syndrome umbilical cords. Quantitative changes in ECM composition and in particular in HA amount might be an explanation for the dynamic course of the nuchal translucency thickness with advancing gestational age. Longitudinal studies performed on weekly measurements by sonography of nuchal translucency (both in trisomy 21 and in euploid fetuses) have shown that in most cases nuchal translucency is a transient phenomenon (Pajkrt et al., 1998; Zoppi et al., 2003). If the cause of the nuchal translucency enlargement is the swelling of interstitial spaces due to the hydrophilic properties of HA, it is reasonable to hypothesize that a decreasing amount of this polysacchar- 
1

WHARTON'S JELLY

HAS 2

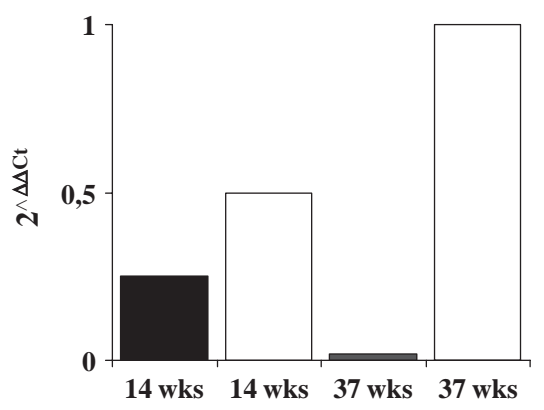

3

WHARTON'S JELLY

HYAL 2

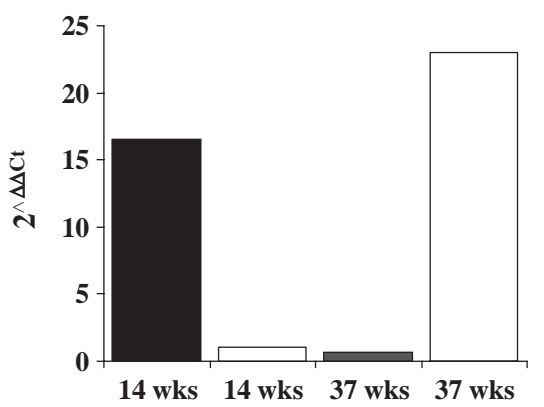

2

UMBILICAL VESSELS

HAS 2

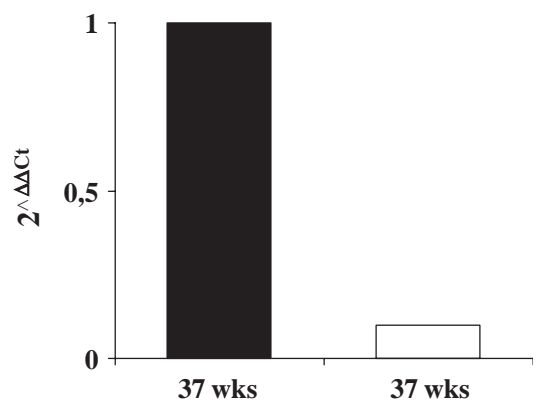

4

UMBILICAL VESSELS

HYAL 2

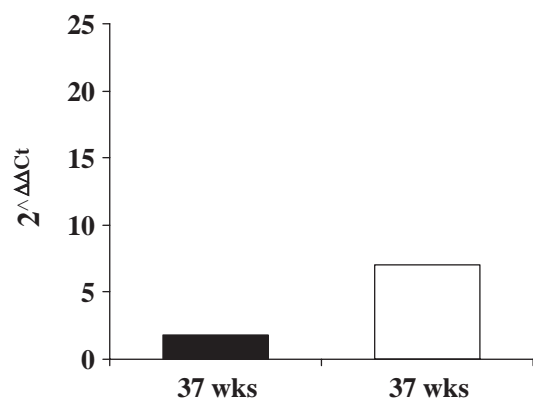

Fig. 6. Gene expression analysis by real-time PCR for hyaluronan synthases 2 (HAS 2) (panels 1 and 2) and hyaluronidases 2 (HYAL 2) (panels 3 and 4); black bars represent euploid specimens white bars represent Down syndrome specimens. The sample with the lower expression of the target was used as calibrator (HAS 2 and HYAL 2 of the umbilical cord vessels from the euploid fetus). $2^{\wedge \Delta \Delta C t}$ represents folds of increment.

ide with advancing gestation leads to a progressive resolution of the enlargement.

The identification of genes encoding for HASs and HYALs and studies of animal and human genetics have widely contributed to the recent understanding of the role played by HA during embryogenesis. Three major physiological functions of this molecule seem to be crucial for

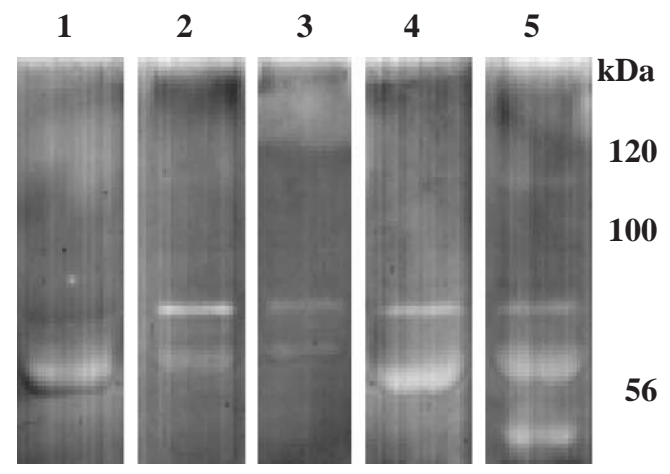

Fig. 7. Hyaluronidase activity by SDS-PAGE zymography using HAcopolymerized gel, developed at $\mathrm{pH} 3.5$ and stained with $0.5 \%$ Alcian blue. Lane 1, positive control (sample from the medium of BC8701 cell line culture); lane 2 and 3, umbilical cord samples of euploid and Down syndrome fetuses at 14 weeks of gestation; lane 4 and 5, umbilical cord samples of euploid and Down syndrome fetuses at term gestation, respectively. normal development: first, HA, due to its peculiar biophysical properties, greatly influences tissue homeostasis and biomechanics; second, the interactions with other components of ECM is essential for the structural integrity of several tissues; third, HA interacts with cell surface receptors influencing cell signaling and function. Particularly, a mechanism by which HA controls cell behavior is the creation of pericellular "coats", a hydrated pericellular zone that facilitates mitosis and cell migration through the extracellular matrix (Toole, 2001; McDonald and Camenisch, 2002). Studies on HA deficient mouse embryos clearly illustrate the importance of each of these functions in morphogenesis (Spicer et al., 2002). Since HA is an ubiquitous glycosaminoglycan, it is reasonable to speculate that an altered composition of ECM in trisomy 21 fetuses may not merely account for the increased nuchal translucency or umbilical cord abnormalities, but could also be involved in the pathogenesis of other clinical features encountered in this chromosomal disease. A high concentration of HA has been shown to impair the migration of neural crest cells, leading to enteric nervous system anomalies (i.e. Hirschsprung's disease, frequently associated with trisomy 21) (Payette et al., 1988) and affecting other organs receiving cell contribution from the neural crest (Kirby et al., 1983). Moreover, studies on HAS 2-/- mice embryos (lacking functional HAS 2 genes) clearly point to the importance of HA in expansion of the 
cardiac cushion matrix and demonstrate that this molecule is required for endothelial cell transformation to mesenchymal cells and their migration into cardiac jelly (Camenisch et al., 2000). Alteration in HA metabolism associated with Down syndrome may therefore be responsible for a specific defect in heart septation, such as the atrioventricular canal (Eisenberg and Markwald, 1995; Camenisch et al., 2000). Possible mechanisms leading to increased content of HA in the extracellular matrix of trisomy 21 fetuses remains to be explored. Overexpression of genes located on chromosome 21 and indirectly involved in HA metabolism (alpha 1 and 2 chain of collagen type VI (Von Kaisenberg et al., 1998b), and the enzyme superoxide-dismutase-SOD (Turrens, 2001)) has been advocated as a possible explanation of the increased HA amount. However, these speculations have never been unequivocally tested and evidences from animal models do not support these possible explanations (Von Kaisenberg et al., 1998a).

The HYAL activity, using zymography, has been widely studied in different cell lines and tissues. In a recent work (Fiszer-Szafarz et al., 2000) it was demonstrated that in human serum and placenta, native HYAL is present and active at $\mathrm{pH} 3.5$ with the most active form of $67 \mathrm{kDa}$. In our study, HYAL activity was observed at $\mathrm{pH}$ 3.5. Two or three different bands were observed which may present different forms of HYAL, with the most active of $67 \mathrm{kDa}$.

Although the results of the present study are not conclusive, the mRNA results on HAS are supported by HA content in the samples and the findings on HYAL 2 have been confirmed measuring the enzymatic activity by zymography. Therefore, the different HAS 2 and HYAL 2 gene expression in Wharton's jelly and umbilical vessels tissues in euploid and Down syndrome UCs is remarkable. Moreover, these findings are in agreement with the different HA amount showed by immunohistochemistry. This suggests that the extracellular matrix of these tissues undergo a diverse regulation of the HA metabolism. The presence of a family of HA with a smaller size in trisomy 21 specimens, as reported by chromatographic analysis, can be explained by the striking imbalance between HAS 2 and HYAL 2 expression, mainly in early gestation.

Cumulatively, our findings imply that the Down syndrome umbilical cord extracellular matrix is affected by a complex alteration of HA metabolism, involving not only its amount, distribution and structure but also cell metabolism. This is particularly true as the umbilical cord's lack of lymphatic network and therefore differences in lymphatic clearance of these polysaccharides may not account for the observed differences in HA content between normal and Down syndrome umbilical cords. Further studies on cell cultures are needed to clarify the HA metabolism in human umbilical cord and the role played by this molecule in the pathogenesis of the different phenotypic features of Down syndrome fetuses.

\section{Experimental procedure}

\subsection{Study population}

Between June 2002 and December 2003, 28 and 13 umbilical cord segments were obtained from euploid and trisomy 21 fetuses, respectively. The karyotype was obtained at chorionic villous sampling, at amniocentesis, from spontaneous abortion tissue or from peripheral neonatal blood. The umbilical cords were collected either after termination of pregnancy, after spontaneous abortion or at delivery. Gestational age was calculated on a reliable recollection of the last menstrual period and confirmed at first trimester ultrasound.

The umbilical cords were processed only if the following conditions were met: 1) known gestational age, 2) singleton gestation, 3) absence of structural malformations in the control population, 4) no histological evidence of funisitis, and 5) three vessel umbilical cord. Medical complications of pregnancy (e.i., preeclampsia, diabetes) were considered exclusion criteria. Immediately after abortion or delivery $1-3$ $\mathrm{cm}$ portions of the umbilical cord were frozen at $-80^{\circ} \mathrm{C}$ and sent to the biochemistry laboratory. The presence of a viable fetus at the time of termination of pregnancy or abortion was a prerequisite for processing the umbilical cord segments. Written informed consent was obtained from all participants to the study. The study was approved by the Institutional Review Board.

\subsection{Reagents used for biochemical analysis}

Proteinase K came from Finnzyme (Epoo, Finland). Hyaluronidase SD and Chondroitinase $\mathrm{ABC}$ were purchased from Seikagaku (Tokyo, Japan), biotinylated HABP was kindly provided by Akira Asari of Seikagaku (Tokyo, Japan). Standard disaccharides were obtained from Sigma (St. Louis, MO, USA), 2-aminoacridone (AMAC) came from Molecular probes (Eugene, OR, USA). FITC-streptavidin was purchased from Vector (Burlingame, CA, USA) and Texas red secondary antibody came from Santa-Cruz Biotechnology (Santa Cruz, CA, USA). Water used for aqueous solutions was of MilliQ ${ }^{\mathrm{TM}}$. All other chemicals were of analytical reagent grade.

\subsection{Isolation and degradation of $H A$}

Frozen samples of human umbilical cords were lyophilized, suspended in $300 \mu \mathrm{l}$ of $0.1 \mathrm{M}$ ammonium-acetate $\mathrm{pH}$ 7 , and digested with protease $\mathrm{K}$ at $60{ }^{\circ} \mathrm{C}$ for $2 \mathrm{~h}$. After enzyme inactivation, $1.2 \mathrm{ml}$ ethanol $96 \%$ (or absolute ethanol) was added and the mixture was frozen at $-20{ }^{\circ} \mathrm{C}$ overnight to precipitate the glycosaminoglycans. After centrifugation at $10,000 \times \mathrm{g}$ at $4{ }^{\circ} \mathrm{C}$ for $15 \mathrm{~min}$, the pellets were solubilized with $100 \mu \mathrm{l}$ of $0.1 \mathrm{M}$ ammonium-acetate $\mathrm{pH} 7$ and sonicated for $1 \mathrm{~min}$ in a ultrasound bath. Polysaccharides were digested with $100 \mathrm{mU} / \mathrm{ml}$ hyalur- 
onidase SD at $37{ }^{\circ} \mathrm{C}$ for $1 \mathrm{~h}$ and then with $100 \mathrm{mU} / \mathrm{ml}$ Chondroitinase $\mathrm{ABC}$ at $37{ }^{\circ} \mathrm{C}$ for $3 \mathrm{~h}$. The samples were lyophilized and derivatized with AMAC, according to a previous study (Calabro et al., 2000).

\subsection{HPLC analysis}

Separation and analysis of derivatized products were performed with a Jasco-Borwin chromatograph system with a fluorophore detector (Jasco FP-920, $\lambda$ ex $=442 \mathrm{~nm}$ and $\lambda \mathrm{em}=520 \mathrm{~nm}$ ). Chromatography was carried out using a reversed phase column $(\mathrm{C}-18,4.6 \times 150 \mathrm{~mm}$, Bischoff, Germany) at room temperature, equilibrated with $0.1 \mathrm{M}$ ammonium acetate, $\mathrm{pH} 7.0$, filtered through a $0.45 \mu \mathrm{m}$ membrane filter. A gradient elution was performed using a binary solvent system composed of $0.1 \mathrm{M}$-ammonium acetate pH 7.0 (eluent A), and acetonitrile (eluent B). The flow rate was $1 \mathrm{ml} / \mathrm{min}$ and the following program was used: pre-run of column with $100 \%$ eluent $A$ for $20 \mathrm{~min}$, isocratic elution with $100 \%$ eluent $\mathrm{A}$ for $10 \mathrm{~min}$ and gradient elution to $30 \%$ eluent B for $30 \mathrm{~min}$. Sample peaks were identified and quantified comparing the fluorescence spectra with standard unsaturated disaccharides, using a Jasco-Borwin software.

\subsection{FPLC analysis of HA molecular size}

Separation and analysis of HA after derivatization with AMAC was performed using Fast Protein Liquid Chromatography (FPLC) (AKTA Amersham Pharmacia Biotech, Uppsala, Sweden). Gel chromatography was carried out using a Superdex ${ }^{\text {TM }} 200$ HR 10/30 prepacked column (10 mm internal diameter, $30-31 \mathrm{~cm}$ height of the packed bed, Amersham Pharmacia Biotech, Uppsala, Sweden) at room temperature. The buffer used was $0.1 \mathrm{M}$ ammonium acetate, $\mathrm{pH} 7.0$, with $0.05 \%$ Tween-20, and the flow rate was $0.35 \mathrm{ml} /$ min. Sample peaks were identified and quantified comparing the absorbance at $280 \mathrm{~nm}$ with standard HA proteins. The identification and quantification of polysaccharides was performed after fraction collection $(2 \mathrm{ml}$ each) and digestion with hyaluronidase $\mathrm{SD}$ and chondroitinase $\mathrm{ABC}$ were as described above. The obtained unsaturated disaccharides were derivatized with AMAC and analysed by FACE (Karousou et al., 2004).

\subsection{HA staining with biotin-labeled HA-binding protein (bHABP)}

Specimen of fixed human umbilical cords from control and trisomy 21 fetuses were prepared for bHABP staining on superfrost slides of $5 \mu \mathrm{m}$ thickness. bHABP recognizes HA saccharidic sequences and is able to localize HA in tissues. The blocking procedure was carried out using 3\% fetal bovine serum (FBS) in PBS buffer for $1 \mathrm{~h}$ at room temperature. The bHABP was diluted in $3 \%$ FBS in PBS to $10 \mu \mathrm{g} / \mathrm{ml}$ and incubated in a humidified chamber at $4{ }^{\circ} \mathrm{C}$ overnight. After washing procedure in PBS, the bHABP was evidentiated with streptavidin conjugate with FITC and visualized using a microscope (Leica, Germany) equipped with an appropriate filter and a digital camera. Control experiments were carried out by incubating the slides with bHABP after digestion with hyaluronidase from Streptomyces at $37^{\circ} \mathrm{C}$ for $2 \mathrm{~h}$.

\subsection{RNA extraction and cDNA synthesis}

Studies were performed on Wharton's jelly and vessels taken separately only from two umbilical cords of euploid fetuses and two Down syndrome fetuses at term of gestation. Wharton's jelly of each umbilical cord was carefully separated from vessels using microsurgery tools and a stereomicroscope. In the specimens of 14th weeks of gestation only the Wharton's jelly was purified for mRNA study. Total mRNA from each sample was isolated using the RNAqueus-4PCR kit from Ambion (Cambridgeshare, UK), followed by a DNase treatment, included in the kit. The concentration of purified RNA was determined fluorometrically using the RiboGreen ${ }^{\circledR}$ RNA Quantitation Reagent and Kit from Molecular Probes (Leiden, the Netherlands). Equal amounts (80 ng) of total RNA from each tissue samples were reverse transcribed using random primers (hexamers), according to the protocol of High Capacity cDNA Archive Kit from Applied Biosystems (Foster City, CA). A RNase inhibitor (Applied Biosystems, Foster City, CA) at final concentration of $1.0 \mathrm{U} / \mathrm{ml}$, was also included in the reaction.

\subsection{Real-time PCR}

Real-time PCR was performed by means of TaqMan technology and ABI Prism 7700 apparatus (Applied Biosystems, CA, USA). All mammalian HASs (HAS1, HAS2 and HAS3) and the more common mammalian HYALs (HYAL 1 and 2) gene expression were studied. RNase $\mathrm{P}$ was used as a housekeeping gene. For these experiments, oligonucleotide primers and TaqMan ${ }^{\circledR}$ probes were Assays-on-Demand of Applied Biosystems. PCR reaction mix contained $2.5 \mu \mathrm{l}$ of cDNA, $12.5 \mu$ l of Universal Master Mix (Applied Biosystems), $1.25 \mu \mathrm{l}$ of Assay-onDemand primer and probe and $8.75 \mu \mathrm{l}$ of nuclease-free water. The PCR program consisted of an initial hot start at $50{ }^{\circ} \mathrm{C}$ for $2 \mathrm{~min}$, followed by $95{ }^{\circ} \mathrm{C}$ for $10 \mathrm{~min}$ and 45 amplification cycles $\left(95^{\circ} \mathrm{C}\right.$ for $15 \mathrm{~s}$ and $60{ }^{\circ} \mathrm{C}$ for $\left.60 \mathrm{~s}\right)$.

For each sample, ABI PRISM 7000 Sequence Detection System (SDS) software plotted an amplification curve by relating the fluorescence signal intensity $(\Delta \mathrm{Rn})$ to the cycle number. A relative quantitative analysis was performed, using the $2^{\wedge(\Delta \Delta \mathrm{Ct})}$ value, where $\Delta \mathrm{Ct}=\mathrm{Ct}$ (target) $-\mathrm{Ct}$ (endogenous control) and $\Delta \Delta \mathrm{Ct}=\Delta \mathrm{Ct}$ (sample) $-\Delta \mathrm{Ct}$ (calibrator) (being $\mathrm{Ct}=$ number of cycle of exponential fluorescence beginning). RNase $\mathrm{P}$ was used as an endogenous control and the sample with the lower expression of the target was used as calibrator. 


\subsection{Protein quantification and zymography}

A 200-300 mg wet-weight piece of umbilical cords of euploid and Down syndrome fetuses at term gestation were homogenised in $1 \mathrm{ml}$ of PBS containing $0.1 \%$ Triton X-100 with the ULTRA-TURRAX ${ }^{\circledR}$. After centrifugation (15 min at $4{ }^{\circ} \mathrm{C}$ ), the supernatants were kept in $-20{ }^{\circ} \mathrm{C}$ until any experimental procedure. Total protein quantification was performed by Bradford method using $3 \mu \mathrm{l}$ of volume of each sample.

The hyaluronidase activity was detected by zymography, as described before by Miura et al., 1995. Briefly, an electrophoresis run was performed in $8 \%$ SDS acrylamide gel (1-mm thickness) containing $170 \mu \mathrm{g} / \mathrm{ml}$ of umbilical cord HA (final concentration). After the electrophoretic run and 2,5\% Triton X-100 incubation, gels were incubated overnight at $37{ }^{\circ} \mathrm{C}$ in $0.1 \mathrm{M}$ sodium formate, $0.15 \mathrm{M} \mathrm{NaCl}, \mathrm{pH} 3.5$ buffer, or in $50 \mathrm{mM}$ citric acid- $\mathrm{Na}_{2} \mathrm{HPO}_{4}, 0.15 \mathrm{M} \mathrm{NaCl}, \mathrm{pH}$ 5.0 buffer. Finally, gels were stained in $0.5 \%$ Alcian blue. HAase activity was detected as unstained bands corresponding to the migrated positions of the enzymes. The medium of cell culture of the BC8701 cell line was used as positive control and a commercial mix of proteins (BIO-RAD, Hercules, CA).

\subsection{Statistical analysis}

Statistical analysis was performed with GraphPad Prism version 3.00 for Windows, (GraphPad Software, San Diego CA). To allow a meaningful comparison of the umbilical cord HA content, for each trisomy 21 specimen a gestational age-matched healthy specimen ( \pm 4 days) was looked for. Statistical significance was considered achieved when $P$ was less than 0.05 .

\section{Acknowledgments}

This work was funded by University of Insubria $(\ll$ Progetto d'eccellenza $\gg$ and FAR) and by MUIR (COFIN prot 2002054473-003) to GDL.

The authors are in debt to Mrs Paola Moretto for her technical assistance during the experimental procedures. The authors are grateful to "Centro Grandi Strumenti dell'Università dell'Insubria” for instruments availability.

\section{References}

Bohlandt, S., von Kaisenberg, C.S., Wewetzer, K., Christ, B., Nicolaides, K.H., Brand-Saberi, B., 2000. Hyaluronan in the nuchal skin of chromosomally abnormal fetuses. Hum. Reprod. 15, 1155-1158.

Brand-Saberi, B., Floel, H., Christ, B., Schulte-Vallentin, M., Schindler, H., 1994a. Alterations of the fetal extracellular matrix in the nuchal oedema of Down's syndrome. Anat. Anz. 176, 539-547.

Brand-Saberi, B., Epperlein, H.H., Romanos, G.E., Christ, B., $1994 b$. Distribution of extracellular matrix components in nuchal skin from fetuses carrying trisomy 18 and trisomy 21. Cell Tissue Res. 277, $465-475$.

Calabro, A., Hascall, V.C., Midura, R.J., 2000. Adaptation of FACE methodology for microanalysis of total hyaluronan and chondroitin sulfate composition from cartilage. Glycobiology 10, 283-289.

Camenisch, T.D., Spicer, A.P., Brehm-Gibson, T., Biesterfldt, J., Augustine, M.L., Calabro Jr., A., Kubalak, S., Klewer, S.E., McDonald, J.A., 2000. Disruption of hyaluronan synthase-2 abrogates normal cardiac morphogenesis and hyaluronan-mediated transformation of epithelium to mesenchyme. J. Clin. Invest. 106, 349-360.

Eisenberg, L.M., Markwald, R.R., 1995. Molecular regulation of atrioventricular valvuloseptal morphogenesis. Circ. Res. 77, 1-6.

Fiszer-Szafarz, B., Litynska, A., Zou, L., 2000. Human hyaluronidases: electrophoretic multiple forms in somatic tissues and body fluids. Evidence for conserved hyaluronidase potential $N$-glycosylation sites in different mammalian species. J. Biochem. Biophys. Methods 45, $103-116$.

Karousou, E.G., Militsopoulou, M., Porta, G., De Luca, G., Hascall, V.C., Passi, A., 2004. Polyacrylamide gel electrophoresis of fluorophore-labeled hyaluronan and chondroitin sulfate disaccharides. Application to the analysis in cells and tissues. Electrophoresis 25, 2919-2925.

Kirby, M.L., Gale, T.F., Stewart, D.E., 1983. Neural crest cells contribute to normal aorticopulmonary septation. Science 220, 1059-1061.

Knudson, C.B., Knudson, W., 2001. Cartilage proteoglycans. Semin. Cell Dev. Biol. 12 (2), 69-78.

Laurent, T.C., 1998. The Chemistry, Biology and Medical Applications of Hyaluronan and Its Derivatives. Wenner-Green Int. Ser., vol. 72. Portland Press, London.

McDonald, J.A., Camenisch, T.D., 2002. Hyaluronan: genetic insights into the complex biology of a simple polysaccharide. Glycoconj. J. 19, $331-339$.

Meyer, K., Palmer, J.W., 1934. The polysaccharide of the vitreous humor. J. Biol. Chem. 107, 629-634.

Miura, R.O., Yamagata, S., Miura, Y., Harada, T., Yamagata, T., 1995. Analysis of glycosaminoglycan-degrading enzymes by substrate gel electrophoresis (Zymography). Anal. Biochem. 225, 333-340.

Moessinger, A.C., Mills, J.L., Harley, E.E., Ramakrishnan, R., Berendes, H.W., Blanc, W.A., 1986. Umbilical cord length in Down's syndrome. AJDC 140, 1276-1277.

Pajkrt, E., De Graaf, I.M., Mol, B.W.J., van Lith, J.M.M., Bleker, O.P., Bilardo, C.M., 1998. Weekly nuchal translucency measurements in normal fetuses. Obstet. Gynecol. 91, 208-211.

Payette, R.F., Tennyson, V.M., Pomeranz, H.D., Pham, T.D., Rothman, T.P., Gershon, M.B., 1988. Accumulation of components of basal laminae: association with the failure of neural crest cells to colonize the presumptive aganglionic bowel of Is/Is mutant mice. Dev. Biol. 125, $341-360$.

Raio, L., Ghezzi, F., Cromi, A., Cereda, E., Passi, A., 2004. Sonographic morphology and hyaluronan content of umbilical cords of healthy and Down syndrome fetuses in early gestation. Early Hum. Dev. 77, 1-12.

Salustri, A., Yanagishita, M., Hascall, V.C., 1990. Mouse oocytes regulate hyaluronic acid synthesis and mucification by FSH-stimulated cumulus cells. Dev. Biol. 138, 26-32.

Spicer, A.P., Tien, J.L., Joo, A., Bowling Jr., R.A., 2002. Investigation of hyaluronan function in the mouse through targeted mutagenesis. Glycoconj. J. 19, 341-345.

Toole, B.P., 2001. Hyaluronan in morphogenesis. Semin. Cell Dev. Biol. $12,79-87$.

Turrens, J.F., 2001. Increased superoxide dismutase and Down's syndrome. Med. Hypotheses 56, 617-619.

von Kaisenberg, C.S., Krenn, V., Ludwig, M., Nicolaides, K.H., BrandSaberi, B., 1998a. Morphological classification of nuchal skin in human fetuses with trisomy 21,18 , and 13 at $12-18$ weeks and in a trisomy 16 mouse. Anat. Embryol. (Berl) 197, 105-124. 
von Kaisenberg, C.S., Brand-Saberi, B., Christ, B., Valian, S., Farzaneh, F., Nicolaides, K.H., 1998b. Collagen type VI gene expression in the skin of trisomy 21 fetuses. Obstet. Gynecol. 91, 319-323.

von Kaisenberg, C.S., Prols, F., Nicolaides, K.H., Maass, N., MeinholdHeerlein, I., Brand-Saberi, B., 2003. Glycosaminoglycans and proteoglycans in the skin of aneuploid fetuses with increased nuchal translucency. Hum. Reprod. 18, 2544-2561.
Weissman, B., Meyer, K., 1954. The structure of hyalobiuronic acid and of hyaluronic acid from umbilical cord. J. Am. Chem. Soc. 76, $1753-1757$.

Zoppi, M.A., Ibba, R.M., Floris, M., Manca, F., Ariana, C., Monni, G., 2003. Changes in nuchal translucency thickness in normal and abnormal karyotype fetuses. BJOG 110, 584-588. 